Int. J. Contemp. Math. Sci., Vol. 1, 2006, no. 9, 409-412

\title{
Construction of singular hypersurfaces and linkage over a finite field
}

\author{
E. Ballico ${ }^{1}$ \\ Dept. of Mathematics, University of Trento \\ 38050 Povo (TN), Italy \\ ballico@science.unitn.it
}

\begin{abstract}
Here we prove two existence theorem over $\mathbb{F}_{q}$ : existence of hypersurfaces with prescribed isolated singularities and existence of "smooth" linkage.

Mathematics Subject Classification: 14J25; 14J70; 14N05; 12E20; 14B05; 14B25

Keywords: singular hypersurface; singular surface; hysurfaces over a finite field; isolated singularity; liaison; linkage
\end{abstract}

\section{The STATEMENTS}

Here we consider two existence theorems over $\mathbb{F}_{q}$. The corresponding constructions are obvious over $\bar{F}_{q}$ and the aim is just to find a relatively low prime power $q$ such that the same constructions may be done over $\mathbb{F}_{q}$. For any $P \in \mathbf{P}^{n}\left(\bar{F}_{q}\right)$ and any integer $m>0$ let $m P$ denote the infinitesimal neighborhood of order $m-1$ of $P$ in $\mathbf{P}^{n}$. Set $0 P=\emptyset$. In section 2 we will study the case of hypersurfaces with prescribed isolated singularities and prove the following result.

Theorem 1. Fix a prime power $q$, an integer $n \geq 2$, an integer $d>0$, an integer $s$ such that $1 \leq s \leq\left(q^{n+1}-1\right) /(q-1)$, integers $m_{i}>0$, and $s$ distinct points $P_{1}, \ldots, P_{s} \in \mathbf{P}^{n}\left(\mathbb{F}_{q}\right)$. Let $Z:=\cup,_{i=1}^{s} m_{i} P_{i}$ and assume $h^{1}\left(\mathbf{P}^{n}, \mathcal{I}_{Z}(d-1)\right)=0$. Set $\delta:=d^{n}-\sum_{1=1}^{s} m_{i}^{n}$ and $\delta_{i}:=m_{i}^{n-1}$. Assume $q \geq(\delta-1) \delta^{n}$. Then there exists a degree $d$ hypersurface $X \subset \mathbf{P}^{n}$ defined over $\mathbb{F}^{q}$ and such that $\operatorname{Sing}(X) \subseteq\left\{P_{1}, \ldots, P_{s}\right\}, P_{i} \in$ $\operatorname{Sing}(X)$ if and only if $m_{i} \geq 2$, and $X$ has multiplicity $m_{i}$ at each $P_{i}$. Furthermore, if $q \geq(\delta-1) \delta^{n}+\sum_{i=1}^{s}\left(\delta_{i}-1\right) \delta_{i}^{n-1}$, then we may find $X$ such that $X$ has an ordinary multiple point with multiplicity $m_{i}$ at $P_{i}$, i.e. the tangent cone of $X$ at $P_{i}$ is a cone over a smooth degree $m_{i}$ hypersurface of $\mathbf{P}^{n-1}$.

When $P_{1}, \ldots, P_{s} \in \mathbf{P}^{n}\left(\bar{F}_{q}\right), P_{i} \notin \mathbf{P}^{n}\left(\mathbb{F}_{q}\right)$ for some $i$, but the set of all pairs $\left\{\left(P_{1}, m_{1}\right), \ldots,\left(P_{s}, m_{s}\right)\right\}$ is invariant for the natural action of the absolute Galois group of $\mathbb{F}_{q}$ we are able to prove the following result.

Theorem 2. Fix a prime power $q$, an integer $n \geq 2$, an integer $d>0$, an integer $s$ such that $1 \leq s \leq\left(q^{n+1}-1\right) /(q-1)$, integers $m_{i}>0$, and $s$ distinct points $P_{1}, \ldots, P_{s} \in \mathbf{P}^{n}\left(\overline{\mathbb{F}}_{q}\right)$. Let $Z:=\cup{ }_{i=1}^{s} m_{i} P_{i}$ and assume $h^{1}\left(\mathbf{P}^{n}, \mathcal{I}_{Z}(d-1)\right)=0$. Assume that the scheme $Z$ and the inclusion of $Z$ in $\mathbf{P O}^{n}$ are defined over $\mathbb{F}_{q}$, i.e. assume that the absolute Galois group of $\mathbb{F}_{q}$ acts trivially on the set of pairs

\footnotetext{
${ }^{1}$ The author was partially supported by MIUR and GNSAGA of INdAM (Italy).
} 
$\left\{\left(P_{1}, m_{1}\right), \ldots,\left(P_{s}, m_{s}\right)\right\}$. Set $\delta:=d^{n}-\sum_{1=1}^{s} m_{i}^{n}$ and $\delta_{i}:=m_{i}^{n-1}$. Assume $q \geq$ $(\delta-1) \delta^{n}$. Then there exists a degree $d$ hypersurface $X \subset \mathbf{P}^{n}$ defined over $\mathbb{F}^{q}$ and such that $\operatorname{Sing}(X) \subseteq\left\{P_{1}, \ldots, P_{s}\right\}, P_{i} \in \operatorname{Sing}(X)$ if and only if $m_{i} \geq 2$, and $X$ has multiplicity $m_{i}$ at each $P_{i}$. Furthermore, if $q \geq(\delta-1) \delta^{n}+\sum_{i=1}^{s}\left(\delta_{i}-1\right) \delta_{i}^{n-1}$, then we may find $X$ such that $X$ has an ordinary multiple point with multiplicity $m_{i}$ at $P_{i}$, i.e. the tangent cone of $X$ at $P_{i}$ is a cone over a smooth degree $m_{i}$ hypersurface of $\mathbf{P}^{n-1}$.

Remark 1. Take $Z$ as in the statements of Theorems 1 and 2 and let $\mu$ be the first integer $t \geq-1$ such that $h^{1}\left(\mathbf{P}^{n}, \mathcal{I}_{Z}(t)\right)=0$. Thus $h^{1}\left(\mathbf{P}^{n}, \mathcal{I}_{Z}(t)\right)=0$ for all $t \geq \mu$ and $d \geq \mu+1$. It is classical that $\mu \leq m_{1}+\cdots+m_{s}-1$ and that we have equality if and only if the points $P_{1}, \ldots, P_{s}$ are collinear $([3])$. If the points $P_{1}, \ldots, P_{s}$ are in linearly general position and $m_{1} \geq m_{2} \geq \cdots \geq m_{s}$, then $\mu \leq$ $\max \left\{m_{1}+m_{2}-1,\left(m_{1}+\cdots+m_{s}+n-2\right) / n\right\}([3])$.

Then we will consider a problem of " nice " linkage over $\mathbb{F}_{q}$ (see [2] for general theory).

Theorem 3. Fix integers $n \geq r \geq 2$ and a prime power $q$. Let $C \subset \mathbf{P}^{n}$ a smooth subscheme with pure codimension $r$ defined over $\mathbb{F}_{q}$. Let $\mu$ be the first non-negative integer $z$ such that $h^{i}\left(\mathbf{P}^{n}, \mathcal{I}_{C}(z-i)\right)=0$ for all $i \geq 1$. Fix $r$ integers $t_{1} \geq \cdots \geq t_{r} \geq$ $\mu+1$. Assume $q \geq \sum_{i=1}^{r}\left(t_{i}^{n}-1\right) t_{i}^{n^{2}}$. Then there are degree $t_{i}$ hypersurfaces $A_{i} \subset \mathbf{P}^{n}$ defined over $\mathbb{F}_{q}$ such that $A_{1} \cap \cdots \cap A_{r}$ is a codimension $r$ hypersurface containing $C$, reduced along $C$ and smooth outside $C$

In the statement of Theorem 3 we do not assume that $C$ is connected or that it is geometrically connected. If $C$ is not geometrically connected we do not assume that all the irreducible components of $C\left(\overline{\mathbb{F}}_{q}\right)$ are defined over $\mathbb{F}_{q}$.

\section{THE PROOFS}

Proof of Theorem 1. Since $\operatorname{dim}(Z)=0$ we have $h^{j}\left(\mathbf{P}^{n}, \mathcal{I}_{Z}(t)\right)=0$ for all $t \in \mathbb{Z}$ and all $j$ such that either $j \geq 2$ and $t \geq-n$ or $2 \leq j \leq n-1$. Let $\mu$ be the first integer $t \geq-1$ such that $h^{1}\left(\mathbf{P}^{n}, \mathcal{I}_{Z}(t)\right)=0$. Thus $h^{1}\left(\mathbf{P}^{n}, \mathcal{I}_{Z}(t)\right)=0$ for all $t \geq \mu$ and $d \geq \mu+1$. By Castelnuovo-Mumford's lemma the homogeneous ideal of $Z$ is generated by forms of degree at most $\mu+1$ and hence it is generated by forms of degree at most $d$. Let $v: M \rightarrow \mathbf{P}^{n}$ be the blowing-up of $\mathbf{P}^{n}$ at the points $P_{1}, \ldots, P_{s}$. We have $R_{*}^{j}\left(\mathcal{O}_{M}\right)=0$ for all $j \geq 1$ and $v_{*}\left(\mathcal{O}_{M}\right)=\mathcal{O}_{\mathbf{P}^{n}}$. Set $E_{i}:=v^{-1}\left(P_{i}\right)$. Hence $E_{i}, 1 \leq i \leq s$. Hence $\operatorname{Pic}(M) \cong \mathbb{Z}^{\oplus s+1}$ and $\operatorname{Pic}(M)$ is freely generated by the classes of the line bundles $v^{*}\left(\mathcal{O}_{\mathbf{P}^{n}}(1)\right)$ and $\mathcal{O}_{M}\left(E_{i}\right), 1 \leq i \leq s$. For all integers $t, z, z_{i} . \quad 1 \leq i \leq z$, set $\mathcal{L}_{t, z}:=v^{*}\left(\mathcal{O}_{\mathbf{P}^{n}}(t)\left(-z E_{1}-\cdots-z E_{s}\right)\right)$ and $\mathcal{L}_{t, z_{1}, \ldots, z_{s}}:=v^{*}\left(\mathcal{O}_{\mathbf{P}^{n}}(t)\left(-z_{1} E_{1}-z \cdots-z_{s} E_{s}\right)\right)$. Since $P_{i} \in \mathbf{P}^{n}\left(\mathbb{F}_{q}\right)$ for all $i, v, M$, each $E_{i}$ and all $\mathcal{L}_{t, z}$ and $\mathcal{L}_{t, z_{1}, \ldots, z_{s}}$ are defined over $\mathbb{F}_{q}$. If $z_{i} \geq 0$ for all $i$, then $v_{*}\left(\mathcal{L}_{t, z_{1}, \ldots z_{s}}=\mathcal{I}_{\cup_{i=1}^{s} z_{i} P_{i}}(t)\right.$.

(a) Here we will check that $R_{*}^{j}\left(\mathcal{L}_{t, z_{1}, \ldots, z_{s}}\right)=0$ for all integers $j, t, z_{1}, \ldots, z_{s}$ such that $j \geq 1$ and $z_{i} \geq 0$ for all $i$. By the projection formula it is sufficient to prove the case $t=0$. The result is true if $z_{i}=0$ for all $i$. Hence we may assume $z_{i}>0$ for some $i$ and use induction on the integer $z_{1}+\cdots+z_{s}$. Hence we may assume that the result is true for the integers $z_{1}, \ldots, z_{i-1}, z_{i}-1, z_{i+1}, \ldots, z_{s}$. Set $B:=\cup_{i=1}^{s} z_{i} E_{i}$ 
and $B^{\prime}:=B-E_{i}$. Thus we have the following exact sequence on $M$ :

$$
0 \rightarrow \mathcal{I}_{B} \rightarrow \mathcal{I}_{B^{\prime}} \rightarrow \mathcal{O}_{E_{i}}\left(B^{\prime}\right) \rightarrow 0
$$

Apply the direct image functor to (1), the cohomology of $E_{i} \cong \mathbf{P}^{n-1}$ and that $\mathcal{O}_{E_{i}}\left(B^{\prime}\right)$ is a degree $z_{i}-1$ line bundle on $E_{i}$.

(b) By part (a) and the definition of $\mu$ we have $h^{j}\left(M, \mathcal{L}_{t, m_{1}, \ldots, m_{s}}\right)=0$ and $h^{0}\left(M, \mathcal{L}_{t, m_{1}, \ldots, m_{s}}\right)=\left(\begin{array}{c}n+t \\ n\end{array}\right)-\sum_{i=1}^{s}\left(\begin{array}{c}m_{i}+n-1 \\ n-1\end{array}\right)$ for all $j \geq 1$, and $t \geq \mu$ and in particular for all $j \geq 1$ and $t \geq d-1$. In the same way we get that $h^{1}\left(M, \mathcal{L}_{t, z_{1}, \ldots, z_{s}}\left(-E_{i}\right)\right)=0$ for all $t \geq \mu+1$.

(c) Here we will show that $\mathcal{L}_{t, m_{1}, \ldots, m_{s}}$ is very ample for all $t \geq \mu+1$ and in particular for $t=d$. It is sufficient to show the surjectivity of the restriction map $\rho_{A, t}: H^{0}\left(M, \mathcal{L}_{t, m_{1}, \ldots, m_{s}}\right) \rightarrow H^{0}\left(A, \mathcal{L}_{t, m_{1}, \ldots, m_{s}}\right)$ for all zero-dimensional subschemes $A \subset M$ such that length $(A)=2$. We distinguish six cases.

(i) $A$ is reduced, say $A=\left\{Q, Q^{\prime}\right\}$ with $Q \neq Q^{\prime}$, and $A \cap\left(E_{1} \cup \cdots \cup E_{s}\right)=\emptyset$;

(ii) $A$ is not reduced and $Q:=A_{\text {red }} \notin E_{1} \cup \cdots \cup E_{s}$;

(iii) $A$ is reduced, say $A=\left\{Q, Q^{\prime}\right\}$ with $Q \neq Q^{\prime}, Q \in E_{i}, Q \in E_{j}$ and $i \neq j$;

(iv) $A$ is reduced, say $A=\left\{Q, Q^{\prime}\right\}$ with $Q \neq Q^{\prime}$, with $Q \in E_{i}$ and $Q^{\prime} \notin E_{1} \cup \cdots \cup E_{s}$;

(v) $A$ is not reduced, $Q:=A_{\text {red }} \in E_{i}$, and $A$ is not contained in $E_{i}$;

(vi) $A \subset E_{i}$ for some $i$.

In cases (i), (ii), (iii), (iv), (v) the morphism $v \mid A: A \rightarrow \mathbf{P}^{n}$ is an embedding. In all these cases it is sufficient to use that the homogeneous ideal of $Z$ is generated by forms of degree at most $t$. Now assume that we are in case (vi). We have $h^{1}\left(\mathbf{P}^{n}, \mathcal{I}_{Z^{\prime}}(t-1)\right)=0$ for all schemes $Z^{\prime} \subset Z$. Take the set-up of part (a) with respect to the integers $z_{j}:=m_{j}$ for all $j$. Apply the twist by $\mathcal{L}_{t, 0, \ldots, 0}$ to the exact sequence (1), use the last vanishing of part (b) and that the line bundle $\mathcal{L}_{d, m_{1}, \ldots, m_{s}} \mid E_{i}$ is the degree $m_{i}$ line bundle on $E_{i} \cong \mathbf{P}^{n-1}$ and hence it is very ample.

(d) By part (c) the line bundle $\mathcal{L}_{d, m_{1}, \ldots, m_{s}}$ is very ample. Notice that we have $\operatorname{deg}\left(\mathcal{L}_{d, m_{1}, \ldots, m_{s}}\right)=d^{n}-\sum_{1=1}^{s} m_{i}^{n}=\delta$. By [1], Th. 1, there is a smooth $W \in$ $\left|\mathcal{L}_{d, m_{1}, \ldots, m_{s}}\right|$. Set $X:=v(W)$. Now we consider the "Furthermore " part. We need to find $W$ as above with the additional property that $W$ is transversal to each $E_{i}$ Since $\operatorname{deg}\left(\mathcal{L}_{d, m_{1}, \ldots, m_{s}} \cap E_{i}\right)=m_{i}, \mathcal{L}_{d, m_{1}, \ldots, m_{s}}$ embedds $E_{i} \cong \mathbf{P}^{n-1}$ by a subsystem of the degree $m_{i}$ Verone embedding. Hence the embedded projective space has degree $m_{i}^{n-1}=\delta_{i}$. Thus its dual variety $\Delta_{i}$ in the projective space $\left|\mathcal{L}_{d, m_{1}, \ldots, m_{s}}\right|$ has degree at most $\left(\delta_{i}\right) \delta_{i}^{n-1}$. The proof of [1], Lemma 1, and our assumption on $q$ implies the existence of a hyperplane of $\left|\mathcal{L}_{d, m_{1}, \ldots, m_{s}}\right|$ transversal to the image of $M$ and to the images of all $E_{i}$.

Proof of Theorem 2. We use the set-up introduced in the proof of Theorem 1. Now some of the line bundles $\mathcal{O}_{M}\left(E_{i}\right)$ may not be defined over $\mathbb{F}_{q}$, but $v, M$ and all line bundles $\mathcal{L}_{t, z}$ are defined over $\mathbb{F}_{q}$. Furthermore for any $t \in \mathbb{Z}$ the line bundle $\mathcal{L}_{t, m_{1}, \ldots, m_{s}}$ is defined over $\mathbb{F}_{q}$. Working over $\bar{F}_{q}$ the proof of Theorem 1 show that $\mathcal{L}_{d, m_{1}, \ldots, m_{s}}$ is very ample. Hence we may again apply [1], Th. 1.

Proof of Theorem 3. Let $v: M \rightarrow \mathbf{P}^{n}$ be the blowing-up of $C$. Since $C$ is smooth, $M$ is smooth. Set $E:=v^{-1}(C)$. For all integers $t$ set $\mathcal{L}_{t}:=v^{*}\left(\left(\mathcal{O}_{\mathbf{P}^{n}}(t)(-E)\right.\right.$. As in the proof of Theorem 1 it is easy to check that $\mathbb{L}_{t}$ is very ample for all $t \geq \mu=1$. we again apply [1], Th. 1. Since a complete intersection in a smooth ambient has no embedded point, to check the existence of $X$ which is reduced along $C$ it is sufficient to test finitely many points of $C\left(\overline{\mathbb{F}}_{q}\right)$. 


\section{REFERENCES}

[1] E. Ballico, An effective Bertini theorem over finite fields, Adv. Geom. 3 (2003), no. 4, 361-363.

[2] J. Migliore, Introduction to liaison theory and deficiency modules, Birkhäuser, Boston, 1998.

[3] N. V. Trung and G. Valla, Upper bounds for the regularity index of fat points, J. Algebra (1995), no. 1, 182-209.

Received: January 30, 2006 